\title{
WSB1: from homeostasis to hypoxia
}

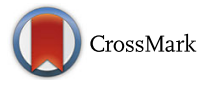

\author{
Moinul Haque ${ }^{1,2,3}$, Joseph Keith Kendal ${ }^{1,2,3}$, Ryan Matthew Maclsaac ${ }^{1,2,3}$ and Douglas James Demetrick ${\text {, } 2,3,4^{*}}^{*}$
}

\begin{abstract}
The wsb1 gene has been identified to be important in developmental biology and cancer. A complex transcriptional regulation of wsb1 yields at least three functional transcripts. The major expressed isoform, WSB1 protein, is a substrate recognition protein within an E3 ubiquitin ligase, with the capability to bind diverse targets and mediate ubiquitinylation and proteolytic degradation. Recent data suggests a new role for WSB1 as a component of a neuroprotective pathway which results in modification and aggregation of neurotoxic proteins such as LRRK2 in Parkinson's Disease, via an unusual mode of protein ubiquitinylation.

WSB1 is also involved in thyroid hormone homeostasis, immune regulation and cellular metabolism, particularly glucose metabolism and hypoxia. In hypoxia, wsb1 is a HIF-1 target, and is a regulator of the degradation of diverse proteins associated with the cellular response to hypoxia, including HIPK2, RhoGDI2 and VHL. Major roles are to both protect HIF-1 function through degradation of VHL, and decrease apoptosis through degradation of HIPK2. These activities suggest a role for wsb1 in cancer cell proliferation and metastasis. As well, recent work has identified a role for WSB1 in glucose metabolism, and perhaps in mediating the Warburg effect in cancer cells by maintaining the function of HIF1. Furthermore, studies of cancer specimens have identified dysregulation of wsb1 associated with several types of cancer, suggesting a biologically relevant role in cancer development and/or progression.

Recent development of an inducible expression system for wsb1 could aid in the further understanding of the varied functions of this protein in the cell, and roles as a potential oncogene and neuroprotective protein.
\end{abstract}

Keywords: WSB1, E3 ubiquitin ligase, Hypoxia, Cancer, HIPK2, VHL

\section{Background}

\section{Discovery and characterization of WSB1}

The E3 ubiquitin ligase, WSB1, has been the subject of many investigations in diverse fields of biology that are finally starting to assemble the spectrum of functional activities attributed to this interesting protein. Aside from its initial characterization in developmental biology, recent studies show WSB1 has an intriguing role in the regulation of thyroid homeostasis, immune responsiveness, glycolysis and hypoxia, as well as potentially influencing the development or growth of cancer. Cell growth, survival and invasion have all been demonstrated to be influenced by WSB1. Recently, WSB1 has been implicated in a potentially important new neuroprotective pathway through its role as a mediator of protein ubiquitinylation.

\footnotetext{
* Correspondence: demetric@ucalgary.ca

'Department of Pathology and Laboratory Medicine, University of Calgary, Calgary, AB T2N 4N1, Canada

2Department of Oncology, University of Calgary, Calgary, AB T2N 4N1, Canada Full list of author information is available at the end of the article
}

\section{Identification and functional definition of wsb1 as a developmental regulator}

ws $b 1$ was originally identified in a virtual homology search by virtue of its relatedness to a large family of suppressor of cytokine signaling (SOCS)-box proteins, along with another related gene $w s b 2$ [15]. The proteins encoded a novel combination of known domains the WD40 repeats structurally located $\mathrm{N}$-terminal to the SOCS box. The chick (wsb1) homologue was discovered as an early marker for limb bud development; likely to be involved in hedgehog pathway signalling [40]. The newly identified gene was initially named $S W i P-1$ (SOCS box and WD repeats in Protein 1). Chick and human WSB1 (SWiP-1) have $88 \%$ protein sequence similarity, while mouse and human WSB1 have $96.29 \%$ sequence similarity $[20,40]$. As such, the protein has been fairly conserved between distinct animal species. Whole mount in situ hybridization revealed that the wsb1 expression pattern in the developing chick closely resembled to that of the hedgehog family of genes, specifically 
sonic hedgehog $(S h h)$. High expression was found in the paraxial mesoderm (somites), limb buds, and other embryonic structures patterned by Shh. It was deduced that positive Shh signalling of wsb1 was from the notochord, as blocking Shh expression in explant cultures depleted wsb1 expression. A negative signal, possibly BMP4, was found from the intermediate and/or lateral mesoderm, preventing local $w s b 1$ expression.

The role of $w s b 1$ has been also studied in zebrafish development. The zebrafish WSB1 ortholog has $75 \%$ protein sequence similarity with human WSB1 [25]. It was noted that $w s b 1$ transcript levels decreased during the mid-blastula transition (MBT) of the zebrafish embryo-an important turnover point of cell cycle regulation and gene expression. Injecting wsb1 mRNA during this critical time resulted in morphological abnormalities and developmental arrest in zebrafish embryos. In summary, WSB1 activity served a significant role in the cell cycle regulation during zebrafish embryogenesis. Expression of the wsbl gene was found to be highly expressed in the intestine, heart, and spleen tissue in the adult zebrafish.

\section{WSB1 gene localization and expression}

The International Radiation Hybrid Mapping Consortium mapped wsb1 to chromosome 17 (G24371) of the human genome, on the $q$ arm in proximity to the centromere (17q.11.1). The gene is 19.5 kilobases long. As reported in Ensembl, the gene encodes for 9 exons, and alternative splicing results in 17 putative transcripts (http://uswest. ensembl.org/Homo_sapiens/Gene/Summary? $\mathrm{db}=$ core;g= ENSG00000109046; $\mathrm{r}=17: 27294076-27315926)$. However, only three major alternative $w s b 1$ transcripts have been reported in the literature $[1,3,36]$. It was shown, through Northern blotting of different human tissues, that the three wsb1 mRNA were highly expressed in the brain, heart, kidney, and placenta [3]. Current review of the literature indicates that only isoform 1 of WSB1 has been detected at the protein level.

Many single nucleotide polymorphisms (SNP) have been identified both within coding and flanking regions of the gene in the human population (http://www.ncbi.nlm.nih. gov/projects/SNP/snp_ref.cgi?showRare $=$ on\&chooseRs=all $\& g o=$ Go\&locusId=26118). A very common SNP (heterozygosity 0.456 , position 363 ) is present within exon 2 . This SNP is a non-synonymous cytosine " $\mathrm{C}$ " to thymine " $\mathrm{T}$ " transition, creating a leucine to serine substitution (L16S) in the expressed protein. Structural biochemistry studies show that the presence of variants of this SNP within the wsb1 RNA results in a difference to its secondary structure [43].

\section{Structure of WSB1}

WSB1 was initially defined by eight WD repeats Nterminal to the C-terminal SOCS box $[15,40]$.

\section{WD domain}

This structural motif, containing approximately 40 amino acids comprised predominantly of tryptophan and aspartate dipeptide repeats, was first identified in bovine transducin $\beta$ [14]. The WD motif defines one of the common domain structures in eukaryote proteins [38]. Structural analysis of the WD domain of $\beta$ transducin showed a 7 -fold propeller-shaped structure with a core of conserved residues and the more variable linker regions displayed on the exterior face of each propeller blade [42]. The non-conserved stretches of amino acids located between $\beta$-strands form the surface of the propeller provide a framework for interactions with other proteins. The WD repeat number within a protein varies, and sometimes can be difficult to define. In the literature, researchers have reported different numbers of WD repeats for WSB1 [41]. Some groups have suggested six WD-repeat domains in WSB1 [44], some seven [10] and some eight $[15,40]$.

\section{SOCS-Box}

The SOCS box domain of WSB1 contains approximately 50 amino acids (1). It may possess a secondary structure composed of three $\alpha$-helices [3, 42] as the region shows homology to that defining the members of the suppressors of cytokine signaling (SOCS) family of proteins [19]. More than 80 proteins containing the SOCS-box domain have been identified [24]. In almost all, including WSB1, the SOCS box can be located at the $C$ terminus [15]. The SOCS box interacts with members of the E3 ubiquitin protein ligase complex such as Elongin $\mathrm{B}, \mathrm{C}$ and the vonHippel Lindau (VHL) tumor suppressor protein [18, 21, 49]. The SOCS-box consists of two main interaction regions-the $\mathrm{BC}$ box and the $\mathrm{Cul}$ box (reviewed in [24]). WSB1 contains the BC box [17]. Elongin $C$ binds strongly to the BC site of the SOCS-box, whereas Elongin B stabilizes these interactions, however does not contact the SOCS-box [5]. Thus SOCS box proteins are important components of the ubiquitin E3 ligase complex.

Unfortunately, to date, the structure of WSB1 has not been determined at high resolution. Structural information of this protein would certainly add more clues with regards to its diversity of functions.

\section{Regulatory functions of WSB1 Known WSB1 regulatory targets in humans}

The first potential function for WSB1 was suggested from the ability of the BC-box containing WSB1 protein to interact with the CUL5 and RBX1 (cullin and ringfinger family members, respectively) proteins to form a potential ubiquitin ligase-E3 complex [17].

Several physiologic targets of WSB1 activity have now been defined, representing different pathways of cellular homeostasis: 
i. Thyroid homeostasis: WSB1 participated in an E3 complex to ubiquitinylate the metabolic regulatory protein, thyroid hormone-activating type 2 iodothyronine deiodinase(D2) [47]. D2 upregulates triiodothyronine (T3) concentration in developing tissues and in the adult brain, thereby controlling several physiological processes [2]. The WD-propeller domain of WSB1 recognized an 18 amino acid loop of D2 [48], while the SOCS domain mediated interaction with the E3 complex [10]. In the developing chick, sonic hedgehog-regulated WSB1 resulted in ubiquitination of D2 and chondrocyte growth, demonstrating a pathway by which sonic hedgehog mediated chick skeletal development [10]. D2 degradation stabilized parathyroid hormone-related peptide (PTHrP) production, an important factor for promoting chondrocyte proliferation [10, 33]. In the rat brain, WSB1 and the D2-deubiquitinase USP-33 expression were restricted to specific regions and worked in opposition to regulate the degradation of D2. Neither gene expression correlated the brain response to thyroid hormone, suggesting that the hormone does not control their regulation [13]. Moreover, correlation between (wsb1) and USP-33 expression existed in other tissues not expressing D2. This could mean other ubiquitinylation substrates in those respected tissues may be similarly regulated by these antagonistic proteins. Recombinant cell line model systems have demonstrated that WSB1 interacts with ubiquitin conjugase UBC-7 in mediating D2 ubiquitination [33].

ii. Immune regulation: WSB1 plays a role in the maturation of the interleukin-21 receptor (IL-21R) [28]. The ligand of IL-21R is interleukin 21 (IL-21), a characterized cytokine with pleiotropic effects. IL-21 is known to regulate lymphocyte and myeloid cell activities. Through interaction with the cytoplasmic regions of IL-21R, WSB1 aided in its maturationallowing for effective glycosylation [28]. By doing so, WSB1 moderated and decreased IL-21R degradation. iii. Metabolic Regulation

a. Hypoxia: wsb1 mRNA expression is upregulated in hypoxia at both mRNA and protein levels in diverse cell lines (Fig. 1a, b). Upregulation of wsb1 is through the activity of HIF-1 [39]. Upregulation of $w s b 1$ can be achieved either through hypoxia chamber treatment or through a hypoxia mimic; such as the addition of desferroximine mesylate (DFX) (Fig. 2a, b), with a corresponding increase in HIF-1 activity [39]. An initial role for WSB1 in hypoxia was first defined through its effect on the regulation of homeodomain-interacting protein kinase 2 (HIPK2) activity [9]. HIPK2 is a member of the nuclear protein kinase family and can induce cell death via the p53 and CtBP-mediated apoptotic pathways when activated $[9,26]$ under conditions such as DNA damage. HIPK2 phosphorylates p53 on S46, which upregulates its pro-apoptotic and cell cycle arrest activities. As such, HIPK2 concentrations are kept low in cells under normal physiological conditions-a process mediated by several proteins. WSB1 with its WD-repeats recognized and bound to HIPK2, which then led to HIPK2 ubiquitination and degradation. The group also demonstrated that WSB1 knockdown by RNA interference increased stability and durability of HIPK2 [9]. Therefore, in unstressed cells, WSB1 is a negative regulator of HIPK2 and is involved in maintaining homeostasis of HIPK2 activity.

Investigation of the potential role of HIPK2 in hypoxia noted the surprising loss of HIPK2 protein. This was not mediated through decreased transcription, but through increased protein degradation. Initial experiments identified the E3-ligase, Siah1, as a mediator of this increased degradation, and further experiments also identified WSB1 as a mediator of hypoxiaassociated HIPK2 degradation [26]. Interestingly, decreased levels of HIPK2 in hypoxia were associated with a decreased level of p53 S46 phosphorylation, and a blunted apoptotic response to the chemotherapeutic drug, adriamycin. These experiments suggest a mechanism by which cancer cells in a hypoxic environment can escape chemotherapy-associated (and maybe radiationassociated) apoptosis through the downregulation of HIPK2 [26]. Recently, VHL (von Hippel Lindau protein), the ubiquitin ligase responsive for targeted degradation of HIF- $1 \alpha$, has also been shown to be a target of WSB1, via functions similar to those mediating degradation of HIPK2 [22]. Thus, activity of WSB1 results in targeted ubiquitination and downregulation of VHL, leading to an increase in HIF-1 activity. The negative regulatory effect of WSB1 on VHL leads to an increase in transcription of HIF-1 targets and stimulates the cellular response to hypoxia, as well as other phenotypes of HIF-1 upregulation [22].

Interestingly, wsb1 disruption during hypoxia through RNAi silencing, or the loss of function of its E3 ubiquitin ligase, were both found to significantly suppress cell migration [6], which also reproduced the findings of other investigators with epithelial cells [22]. Upon further investigation, a strong negative correlation between WSB1 and a Rho-inhibiting protein 


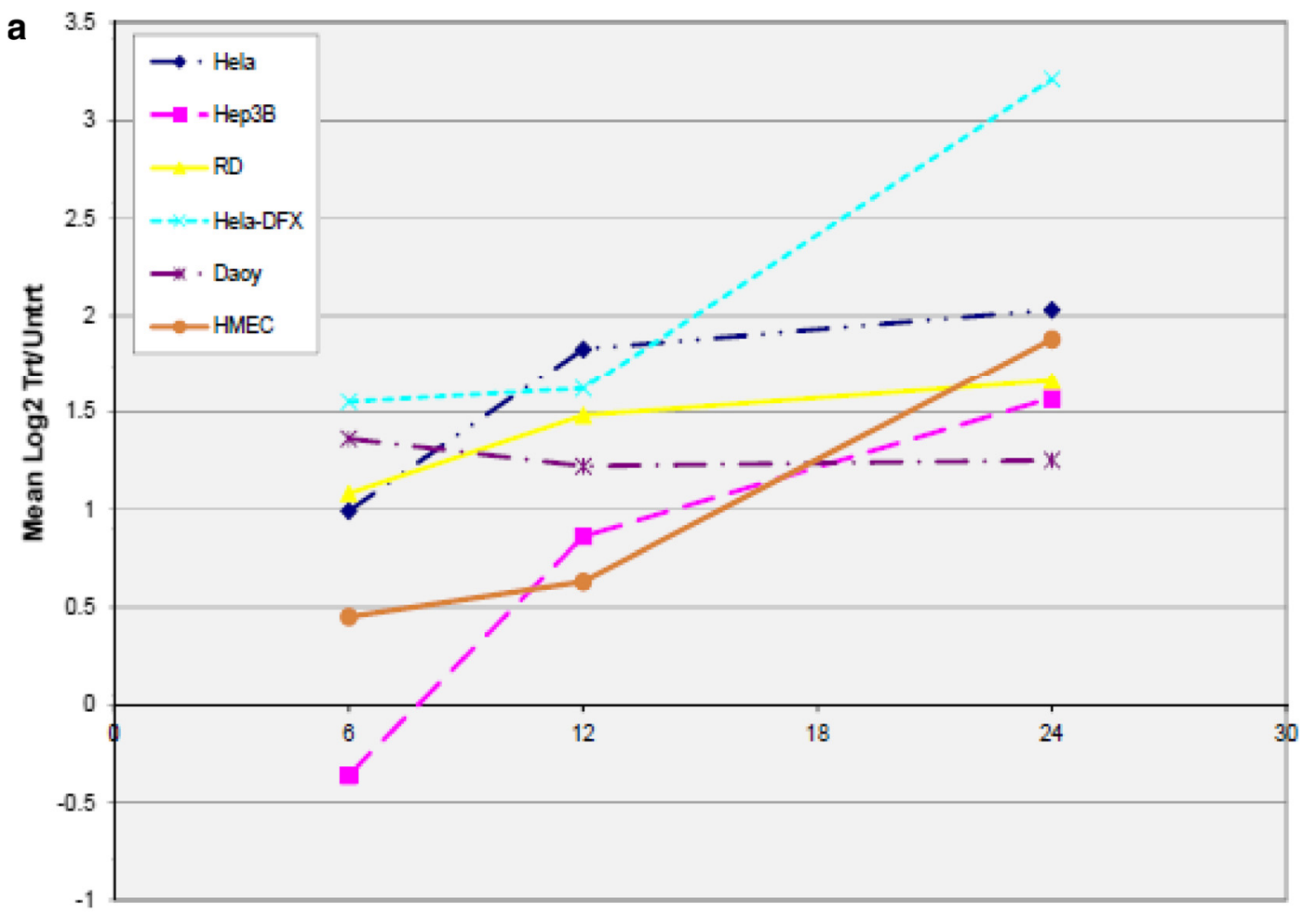

Time (hrs)

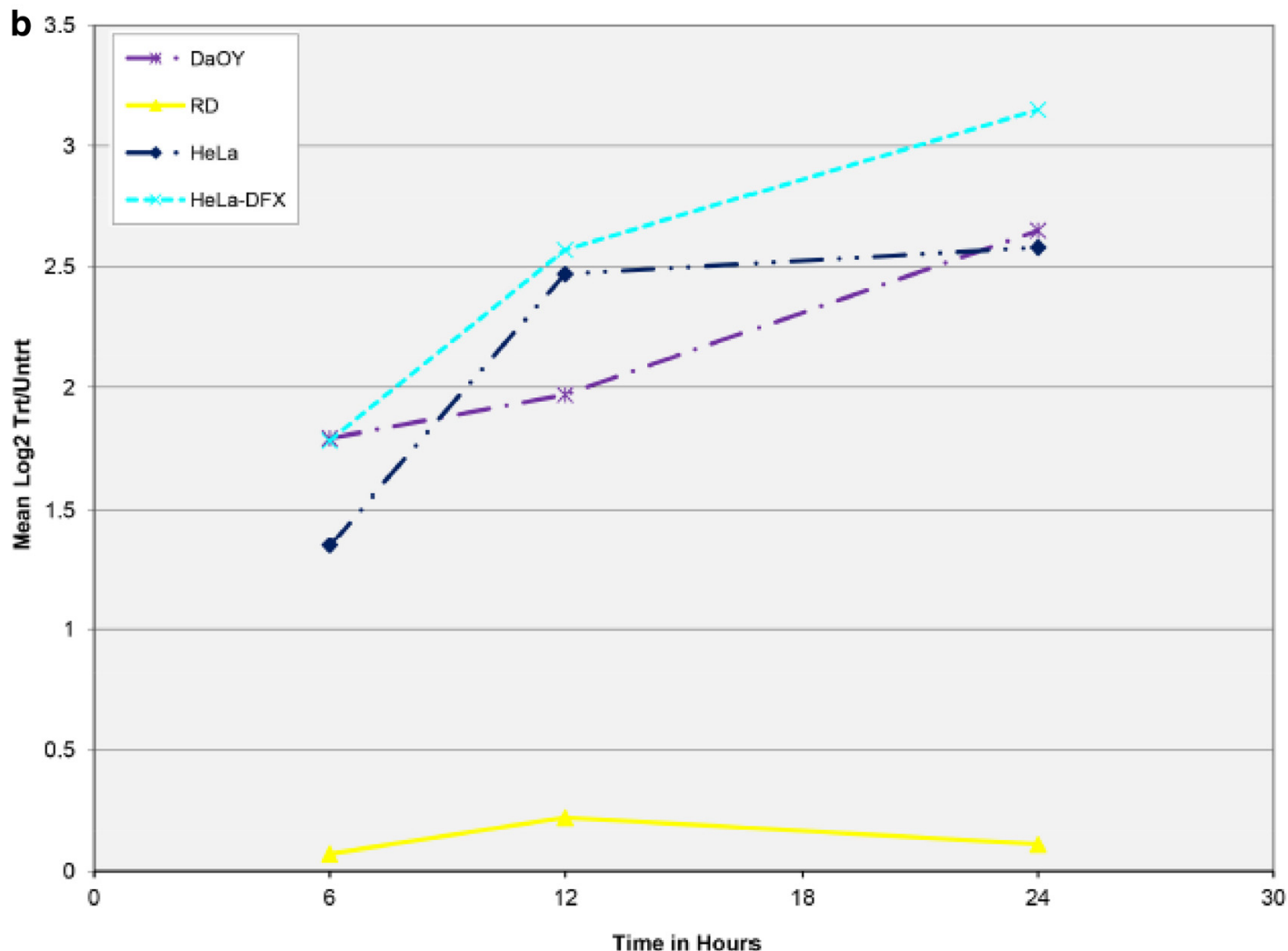

Fig. 1 (See legend on next page.) 
(See figure on previous page.)

Fig. 1 Regulation of WSB1 mRNA Expression by Hypoxia. Various cell lines including HeLa, Hep3B, RD, Daoy, and HMEC were treated with severe hypoxia for various time points (6,12 and $24 \mathrm{~h}$ ) as per methods published previously [34] and total mRNA extracted and evaluated by expression microarray. Panel a shows the normalized $\log _{2}($ Treated/Untreated) ratios of EST AL110269 (wsb1) plotted for each time point. Cell lines are indicated by differing colors/lines. Panel $\mathbf{b}$ shows the normalized $\log _{2}$ (Treated/Untreated) ratios of EST AB018285 (JHDM2A/JMJD1A), a known HIF-1 target [31, 46] plotted for each time point. mRNA levels at Time 0 are normalized to the $\log _{2}$ value "0". Cell lines are indicated by differing colors/lines. For both panels, Hela cells treated with 260uM desferroximine (Hela-DFX) was used as a positive control

(RhoGDI2) levels were found [6]. Functional studies confirmed that WSB1, using its SOCS box, directly ubiquitinylates RhoGDI2 and targets its degradation.

b. Glucose: HIF-1 has long been known to regulate genes involved in glucose metabolism (reviewed in [50]), as well as WSB1, and is a likely mediator of the well known Warburg effect of altered glucose metabolism in cancer cells. Recent work has identified that wsb1, which is a transcriptional target of HIF-1, is also target for the microRNA (miR) miR-592, which downregulates its expression [16]. Interestingly, miR-592 was identified to be commonly downregulated in hepatocellular carcinomas (HCCs), and that tumors with low expression of miR-592 tended to have a poor prognosis. Furthermore, upregulation of miR-592 decreased glucose metabolism in HCC cells and the growth of HCC tumor xenografts, and, conversely, downregulation of miR-592 was associated with increased HCC growth. Specimens of HCC from cancer patients showed a general inverse correlation between miR-592 levels and the levels of WSB1 and HIF1 $\alpha$. As WSB1 is a positive regulator of HIF-1 and miR-592 is a negative regulator of WSB-1, investigators have postulated that miR-592 is a negative mediator of the Warburg effect in HCC cells, and that the HIF-1/WSB1/miR-592 regulatory axis may be a new point of treatment for HCC cases [16].

\section{Protein aggregation}

Several degenerative neurological disorders are characterized by the formation of protein aggregates, forming insoluble complexes or even microscopially visible macroaggregates ("bodies"). More famous examples include the neurofibrillary tangles of Alzheimer's dementia, as well as Lewy bodies, found in the dopaminergic neurons of the substantia nigra of Parkinson's Disease (PD). Recently, one of the proteins commonly found in Lewy bodies, the kinase LRRK2, was found to be a target of WSB1 [30]. LRRK2 is known to be a mediator of a genetic PD syndrome, which is associated with gain of LRRK2 activity as a consequence of an activating mutation. WSB1 E3 ubiguitin ligase activity was shown to result in ubiquitinylation of LRRK2. Interestingly, though, instead of facilitating the conjugation of ubiquitin through the K48 residue, which is associated with proteosomal degradation, WSB1 facilitated the conjugation of ubiquitin residues K27 and K29 to LRKK2. This covalent association of ubiquitin with LRKK2 led to protein aggregation and loss of soluble LRKK2, rather than proteosomal degradation. Immunohistochemical study of Lewy bodies identified both WSB1 and LRRK2 within Lewy bodies associated with LRRK2-associated Parkinson's Disease, but not PD associated with a different etiology. Furthermore, overexpression of $w s b 1$ led to significantly decreased neuronal toxicity due to upregulated LRRK2, while decreased wsb1 expression led to increased neuronal toxicity. The authors hypothesize that these aggregates of insoluble,

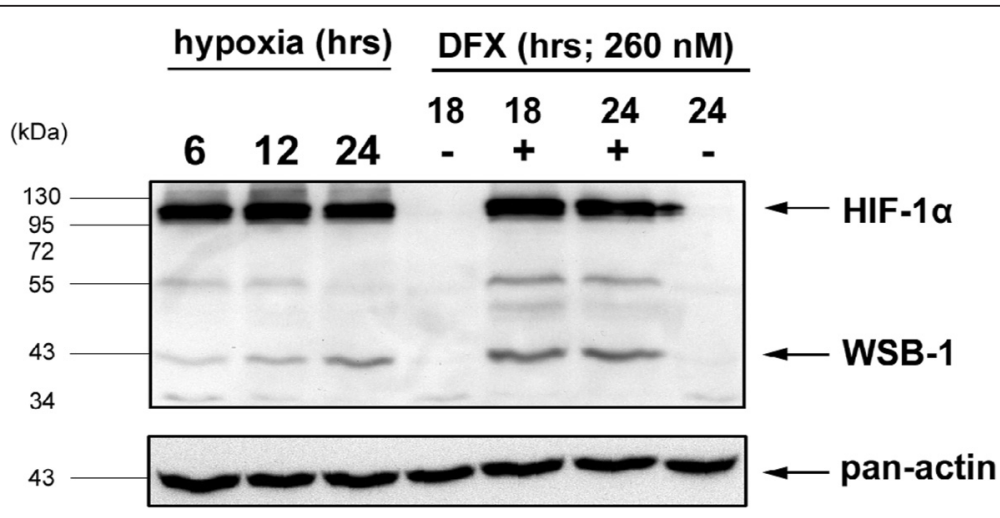

Fig. 2 Regulation of WSB1 Protein Expression by Hypoxia. This figure shows a Western Blot of Hela cells treated with severe hypoxia or 260uM desferroximine for various timepoints (hours as indicated) and stained with either anti-pan-actin, anti-HIF1a or anti-WSB1 as per previously published methods [4]. The untreated (24 h-DFO) specimen is also the same as the Time 0 hypoxia specimen as the experiments were performed simultaneously 
neurotoxic proteins are a cellular protective mechanism mediated by E3 proteins such as WSB1 [30].

\section{WSB1 compared to WSB2}

WSB1 is related to WSB2 with an amino acid sequence similarity of $65 \%$ [15]. Surprisingly, WSB1 and WSB2 have both similar and conflicting functional properties. In contrast to WSB1, WSB2 was found to be a negative regulator of IL-21R expression and IL-21-induced signal transduction [27]. Overexpression of $w s b 2$ reduced IL-21R expression whereas siRNA targeting of $w s b 2$ enhances IL21R expression [27]. Expression of $w s b 2$ was found in mouse embryonic and adult gonads [35]. wsb2 expression persisted only in male embryos, suggesting that it had a male specific role in gonad development [35]. Ihh signalling inhibitors led to a downregulation of $w s b 2$ mRNA. Based on these results, Sarraj et al. (2007) concluded that wsb2 could be a potential consequence of hedgehog signalling similar to wsb1 [35]. (wsb2) has also been found to regulate the expression of the granulocyte colonystimulating factor (G-CSF) receptor. WSB2 binding reduced the proliferative signalling of G-CSF required for neutrophil development [12].

\section{WSB1 and cancer}

As E3 ubiquitin ligases mediate the regulated degradation of various regulatory proteins crucial for cell survival, many are candidate oncoproteins or tumor suppressor proteins. E3 ubiquitin ligases such as MDM2 are known oncoproteins [7], whereas others such as VHL are known tumor suppressor proteins [32]. Classification of which role for the E3 ubiquitin ligase depends on the role of their major target protein. In addition, members of the same family can have either function, such as the F-box family of proteins [45]. Reviews of SOCS-box proteins are complex, due to the diversity of proteins that harbor this motif responsible for interaction with Elongin B and C, but whose target association may be determined by different domains such as WD40 or ankyrin. For example, SH2-containing SOCS proteins (eg. SOCS1-7) likely function as tumor suppressor proteins [11]. In this context, downregulation or decreased function of WSB1 would be expected in cancer cells, however, participation of WSB1 in ubiquitin-mediated degradation of a tumor suppressor gene such as VHL may also demonstrate a role for WSB1 as an oncoprotein.

An interesting mechanism by which WSB1 may contribute to cancer development is through its role as an E3 ligase regulating the previously mentioned HIPK2, as well as RhoGDI2 and VHL during hypoxia [6, 9, 22, 39]. HIF1, RhoGDI2, HIPK2, and miR-592 could be considered to form a regulatory network, linked by WSB1. Regulation of multiple, important regulatory proteins by WSB1 in the cellular response to hypoxia, may also facilitate cancer phenotypes (summarized in Fig. 3). HIF-1, a key regulatory transcription factor of the cellular hypoxia response comprised of HIF- $1 \alpha$ and HIF1- $\beta$ subunits, binds to hypoxia response elements (HRE) in the 5 ' promoter region of the wsb1 gene and induces its expression [39]. Silencing of HIF-1 $\alpha$ by siRNA diminished hypoxiainduced ws $b 1$ expression. Hypoxia also induced significant expression of the $w s b 1$ gene in cancer specimens $[6,8]$. In human hepatocellular carcinoma (HCC) or other cell lines, under hypoxic conditions, WSB1 binds and mediates degradation of HIPK2. This inhibits cancer cell apoptosis associated with anti-cancer treatments [39]. wsb1 silencing through RNA interference in hypoxic HCC cell lines, rescued HIPK2. This change made HCC significantly more prone to death via chemotherapeutic agents [39].

VHL is a known tumor suppressor, which targets HIFs for degradation with its E3 ubiquitin ligase activity. HIFs, however, were not found to be directly targeted by WSB1 E3 ubiquitin ligase activity. Further studies demonstrated that WSB1 is a direct negative regulator of VHL in HEK293 cells [22]. WSB1 ubiquitinated and targeted VHL for degradation. RNAi silencing of wsb1 in cancer cells under normoxia and hypoxia treatment stabilizes VHL, while inhibition of the WSB1 effect with MG132, a proteasome inhibitor, led to VHL survival. WSB1 and VHL were also shown to co-immunoprecipitate. Experimental data suggested that two C-terminal WD-repeats of WSB1 and the SOCS box were essential requirements for VHL ubiquitination. As the degradation of VHL through WSB1 further stabilized HIFs, this resulted in increased expression of wsb1-effectively creating a positive feedback loop. HEK293 cells overexpressing wsb1 demonstrated an increase of HIF-regulated downstream genes and this correlated to increased metastatic potential through expression of VEGFA, ALDOC, CA9, and SAP30. However, as might be expected, ws $b 1$ expression does not significantly increase HIF1 $\alpha$ mRNA levels.

It was recently demonstrated that osteosarcomas in both in vivo and in vitro experiments had increased metastatic potential under hypoxia [6]. Higher invasive potential, as quantitatively deduced by observation of increased cellular migration, were found in two osteosarcoma cell lines and three primary osteoblast lines. A549 and HeLa cell lines were also observed to behave similarly. In each primary cell line, hypoxia upregulated HIF- $1 \alpha$, which subsequently led to downstream wsb1 upregulation. RhoGDI2 downregulation in osteosarcomas has been previously shown to activate the Rac1 and Rho pathway, which can lead to increased tumor cell metastasis potential. Hypoxia-induced WSB1 was associated with downregulation of RhoGDI2, showing physiologic relevance of this interaction in hypoxia.

While hypoxia upregulates HIF-1, and leads to upregulation of many genes manifesting the hypoxic survival 


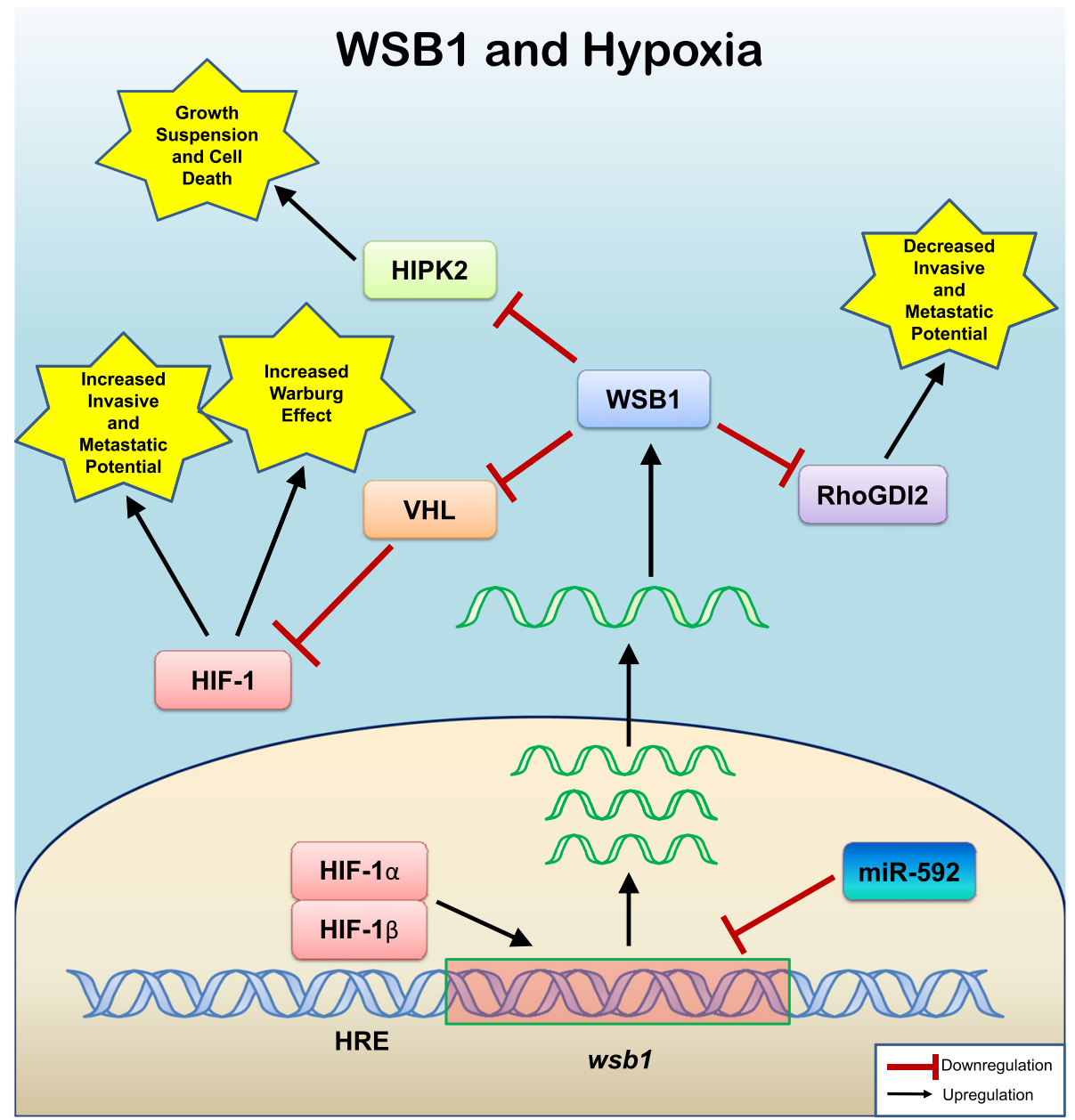

Fig. 3 WSB1 has Diverse Roles in Hypoxia That May Also Drive Cancer Cell Growth. This figure summarizes the varied roles that WSB1 performs in the cellular response to hypoxia in cancer cells. In the normal hypoxic response, low oxygen results in inhibition of the VHL protein, which normally facilitates the degradation of the alpha subunit of the main hypoxia transcription factor HIF-1. HIF-1 upregulates a wide variety of target genes through interaction with its consensus Hypoxia Response Element, including wsb1, and a variety of other genes which can lead to enhanced cellular survival and metastasis in cancer cells. Upregulation of wsb 1 results in enhanced degradation of the VHL protein through WSB1, and increased activity of HIF-1, as well as increased degradation of other WSB1 targets HIPK2 and RhoDGI2, the effects of which can also stimulate cancer cell growth and metastasis. Aside from upregulation of the many HIF-1 target genes associated with the cellular response to hypoxia, HIF-1 also upregulates many of the genes whose products mediate the Warburg effect of glucose metabolism in cancer cells. The microRNA, miR-592, downregulates WSB-1 and thus can modulate the Warburg effect and the response to hypoxia. In summary, recent characterization of diverse roles for WSB1 in the cellular response to hypoxia suggests that WSB1 may function as an oncogene, leading to enhanced cancer cell survival and metastasis through a variety of physiologic roles

response, HIF-1 also likely mediates much of the Warburg effect of altered glucose metabolism in cancer cells [30]. The published evidence now strongly suggests that in hypoxia, WSB1, through diverse mechanisms such as protection of HIF-1 through downregulation of VHL, HIPK2 and/or RhoGDI2, contributes to tumor metastasis $[6,16,22,26]$.

Dysregulation of wsb1 expression has been documented in diverse cancer cell types, including neuroblastoma (NB), hepatocellular carcinoma (HCC), pancreatic, osteosarcoma and breast cancer cells [1, 6, 8, 23, 29, 36, 37, 39]. Copy number changes of $w s b 1$ can be identified in cases of neuroblastoma. Copy number of wsb1 correlated strongly with wsb1 expression [8]. Neuroblastoma cases of stage $4 S$ more frequently showed gains of $w s b 1$ gene copy number than stage 4- cases. Increase of $w s b 1$ gene expression correlated with good outcome, suggesting that wsb1 expression may have a role in the biology of good prognosis of certain neuroblastoma [8]. Through gene expression analysis, wsb1 expression was positively correlated with high metastatic potential in melanoma, pancreatic and urinary bladder cancer cohorts [22]. Similarly, in certain breast and colon cancers, low wsb1 expression was correlated with higher survival. 


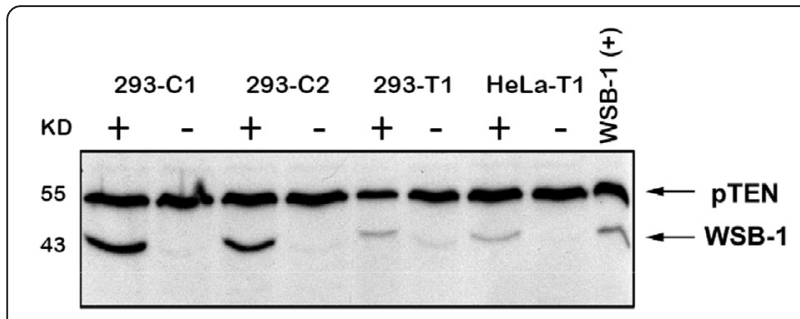

Fig. 4 Preparation of Tet-inducible-WSB1 Cell Lines. Full length wsb1 isoform 1 was prepared by RT-PCR and cloned into a shuttle vector. Clones were sequenced and both SNP variants $C$ and T were identified and each was cloned in frame, into the Tet-On $3 G$ system vector, pTRE3G (Clontech). The wsb1 insert in PTRE3G was sequenced prior to use for transfection. wsb1-pTRE3G was transfected into either HeLa or HEK293 containing a stably transfected TET protein expressing construct (Clontech). Puromycin resistant clones were selected and evaluated for their inducibility with $1 \mathrm{mg} / \mathrm{ml}$ doxycycline. Figure 4 demonstrates the inducibility of wsb1 in the presence or absence of doxycycline. Example cell lines include either WSB1 "C" variant HEK293 (2 clones), or "T" variant (one cell line) or HeLa "T" variant
Two groups of investigators, individually studying pancreatic cancer and neuroblastoma, found that the three wsb1 transcripts had antagonistic functions to each other $[1,36]$. Interestingly, both groups discovered that tumor hypoxia modulated the splice form of the wsb1 mRNA created $[1,36]$. This was identified through the xenotransplantation of respective cancer cells in mouse, and quantifying the wsb1 mRNA in the extract by realtime PCR (qPCR) [1,36]. Interestingly, both groups discovered that in xenograft tumors, wsb1 mRNA encoding for isoform 1 and 2 had been downregulated, whereas the wsb1 mRNA encoding for isoform 3 had been upregulated [1]. Similar results had been obtained when the groups used chemotherapeutic stress agents in in vitro cancer cell cultures $[1,36]$. Silencing expression of the wsb1 transcripts through siRNA in the xenograft tumors showed reduced growth, enhanced apoptosis rate, and increased sensitivity to chemotherapeutic agents. Specifically, these effects were attributed to the expression of $w s b 1$ isoform $3[1,36]$. In support of this view, the retroviral forced expression of $w s b 1$ isoforms 1 and 2 promoted cell growth and increased sensitivity of cells towards apoptosis.

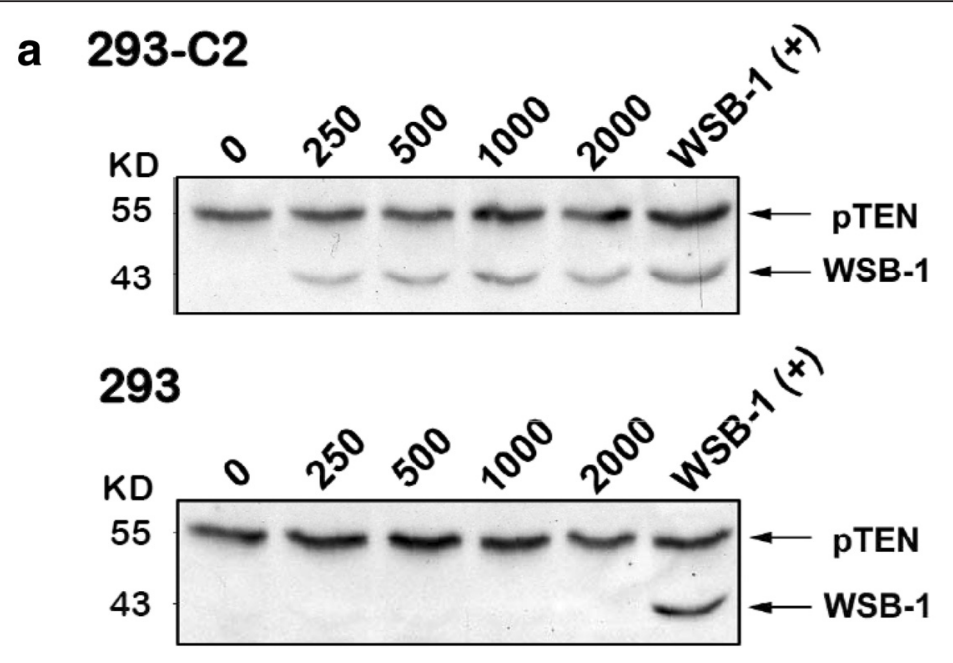

b 293-C2

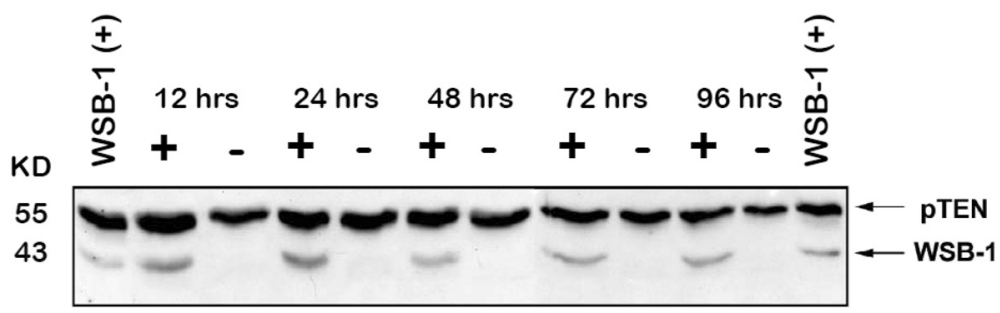

Fig. 5 Characterization of WSB1 Induction with Doxycycline. Panel a HEK293 cells containing the inducible WSB1 "C" construct (293-C2) or native HEK293 cells (293) were treated with various concentrations of doxycycline (250, 500, 1000, $2000 \mathrm{ug} / \mathrm{ml})$ for 24 h, then used for Western blotting with \#604 anti-WSB1 and anti-pTEN (New England Biolabs) primary antibodies followed by detection with anti-rabbit lg secondary antibody. Lysates from Hela cells treated with desferroximine (260 uM) were used as positive controls (WSB-1 (+)). Panel b HEK293 cells containing the inducible WSB1 "C" construct (293-C2) were treated with $1000 \mathrm{ug} / \mathrm{ml}$ doxycycline for various time points (in hours) and then subjected to Western blotting as for Panel A (above). Lysates from Hela cells treated with desferroximine (260 uM) were used as positive controls (WSB-1 (+)) 
In contrast, retroviral forced expression of wsb1 isoform 3 reduced cell proliferation and enhanced resistance to apoptosis [1].

\section{Conclusions}

WSB1 has been identified through diverse investigations and in different experimental model systems as an important regulator of targeted protein destruction or aggregation. Protein targets of WSB1 are involved in cellular processes as diverse as thyroid hormone regulation, immune regulation and regulation of the cellular response to hypoxia. Likely through its latter effects (Fig. 3), WSB1 has been shown to be a likely regulator of abnormal proliferation and metastasis in cancer.

Recently, we have constructed two different, tetracyclineinducible wsb1-expressing cell lines by cloning full length wsb1 into the mCherry expression vector, pTRE3GmCherry (Tet-On 3G, Clontech) and expressing WSB1 in either HeLa or HEK293 cells (Fig. 4). Cell lines were also created using either the "C" or "T" polymorphism variants of wsb1. Tet-inducible wsb1 approximates the levels seen in hypoxia at $24 \mathrm{~h}$ post-induction, using an optimal concentration of doxycycline $(1 \mathrm{mg} / \mathrm{ml}$, Fig. 5). WSB1-inducible cell lines such as these, permit the study of WSB1 induced by doxycycline, without complication by the complex regulatory effects manifested through treatment of cells with hypoxia or hypoxia-mimics, which stimulate pleomorphic effects that are non-specific to WSB1 regulation. Thus, recent development of WSB1-inducible cell lines should facilitate study of this very interesting, potential oncoprotein.

\section{Abbreviations \\ D2, thyroid hormone-activating type 2 iodothyronine deiodinase; DFX, desferroximine meselyate; G-CSF, granulocyte colony stimulating factor; HCC, hepatocellular carcinoma; HIF, hypoxia inducing factor; HIPK, homeodomain- interacting protein kinase; Ihh, Indian hedgehog; LRRK, Leucine-rich repeat kinase; MBT, mid blastula transition; miR, microRNA; mRNA, messenger RNA PD, Parkinson's disease; PTH, parathyroid hormone; PTHrp, parathyroid hormone-related peptide; RhoGDI, Rho guanosine diphosphate dissociation inhibitor; RNA, ribonucleic acid; RNAi, inhibitory RNA; SH2, src homology domain; SNP, single nucleotide polymorphism; SOCS, suppressors of cytokine signaling; VHL, von Hippel-Lindau}

\section{Acknowledgements}

The authors thank Ms. Claude Veillette for very helpful technical assistance. Mr. Bob Winkfein is acknowledged for helpful ideas in the construction of the Tet-On, WSB1 expression vector.

\section{Funding}

$\mathrm{MH}$ acknowledges the support of a University of Calgary Markin USRP Summer Studentship award. RMM and JKK acknowledge the support of their Alberta Innovates Summer Studentship awards. DJD acknowledges operating support from the Ruth Barker Foundation, and salary support from Calgary Laboratory Services.

\section{Availability of data and material}

Most of the data reviewed has been previously published elsewhere. Other data will be available through contact with the corresponding author.

\section{Authors' contributions}

$\mathrm{MH}$ reviewed much of the literature cited, contributed a significant portion of the writing of this manuscript and prepared some of the data and some of the figures. JKK prepared the inducible expression system and prepared some of the data. RMM assisted with the preparation of the inducible expression system and with the preparation of some of the data. DJD conceived the review, reviewed much of the literature, contributed a significant portion to the writing of the manuscript, prepared many of the figures and supervised the experimental work. DJD addressed the reviewers comments and updated the revised manuscript. All authors reviewed the revised manuscript, prior to submission for publication. All authors read and approved the final manuscript.

\section{Authors' information}

$\mathrm{MH}$ is an undergraduate student at the University of Calgary. JKK and RMM are currently surgical and medical residents, respectively, at the University of Calgary.

\section{Competing interests}

The authors declare that they have no competing interests with data presented in this manuscript or with any of the work cited within.

\section{Consent for publication}

All authors have reviewed the final draft of this manuscript and consent to its publication.

Ethics approval and consent to participate

Not applicable.

\section{Author details}

'Department of Pathology and Laboratory Medicine, University of Calgary, Calgary, AB T2N 4N1, Canada. ${ }^{2}$ Department of Oncology, University of Calgary, Calgary, AB T2N 4N1, Canada. ${ }^{3}$ Department of Medical Biochemistry, University of Calgary, Calgary, AB T2N 4N1, Canada. ${ }^{4}$ Calgary Laboratory Services, Room 302, HMRB, 3330 Hospital Dr. N.W., Calgary, AB T2N 4N1, Canada.

Received: 29 March 2016 Accepted: 11 July 2016

Published online: 19 August 2016

\section{References}

1. Archange C, Nowak J, Garcia S, Moutardier V, Calvo EL, Dagorn JC, lovanna $J$. The WSB1 gene is involved in pancreatic cancer progression. PLoS One. 2008;3(6):e2475.

2. Arrojo EDR, Bianco AC. Type 2 deiodinase at the crossroads of thyroid hormone action. Int J Biochem Cell Biol. 2011;43(10):1432-41.

3. Arrojo EDR, Fonseca TL, Werneck-de-Castro JP, Bianco AC. Role of the type 2 iodothyronine deiodinase (D2) in the control of thyroid hormone signaling. Biochim Biophys Acta. 2013;1830(7):3956-64.

4. Box AH, Kim SM, Demetrick DJ. AKT loss in human epithelial cells treated with severe hypoxia. Biochim Biophys Acta. 2010;1803(8):951-9.

5. Bullock AN, Debreczeni JE, Edwards AM, Sundstrom M, Knapp S. Crystal structure of the SOCS2-elongin C-elongin B complex defines a prototypical SOCS box ubiquitin ligase. Proc Natl Acad Sci U S A. 2006;103(20):7637-42.

6. Cao J, Wang Y, Dong R, Lin G, Zhang N, Wang J, Lin N, Gu Y, Ding L, Ying $M, H e$ Q, Yang B. Hypoxia-induced WSB1 promotes the metastatic potential of osteosarcoma cells. Cancer Res. 2015;75(22):4839-51.

7. Chao CC. Mechanisms of p53 degradation. Clinica Chimica Acta. 2014; 438C:139-47.

8. Chen QR, Bilke S, Wei JS, Greer BT, Steinberg SM, Westermann F, Schwab M, Khan J. Increased WSB1 copy number correlates with its over-expression which associates with increased survival in neuroblastoma. Genes Chromosomes Cancer. 2006:45(9):856-62.

9. Choi DW, Seo YM, Kim EA, Sung KS, Ahn JW, Park SJ, Lee SR, Choi CY. Ubiquitination and degradation of homeodomain-interacting protein kinase 2 by WD40 repeat/SOCS box protein WSB-1. J Biol Chem. 2008;283(8):4682-9.

10. Dentice M, Bandyopadhyay A, Gereben B, Callebaut I, Christoffolete MA, Kim BW, Nissim S, Mornon JP, Zavacki AM, Zeold A, Capelo LP, Curcio-Morelli C, Ribeiro R, Harney JW, Tabin CJ, Bianco AC. The Hedgehog-inducible ubiquitin ligase subunit WSB-1 modulates thyroid hormone activation and PTHrP secretion in the developing growth plate. Nat Cell Biol. 2005;7(7):698-705.

11. Elliott J, Hookham MB, Johnston JA. The suppressors of cytokine signalling E3 ligases behave as tumour suppressors. Biochem Soc Trans. 2008;36(Pt 3):464-8. 
12. Erkeland SJ, Aarts LH, Irandoust M, Roovers O, Klomp A, Valkhof M, Gits J, Eyckerman S, Tavernier J, Touw IP. Novel role of WD40 and SOCS box protein-2 in steady-state distribution of granulocyte colony-stimulating factor receptor and G-CSF-controlled proliferation and differentiation signaling. Oncogene. 2007;26(14):1985-94.

13. Fekete C, Freitas BC, Zeold A, Wittmann G, Kadar A, Liposits Z, Christoffolete MA, Singru P, Lechan RM, Bianco AC, Gereben B. Expression patterns of WSB-1 and USP-33 underlie cell-specific posttranslational control of type 2 deiodinase in the rat brain. Endocrinol. 2007;148(10):4865-4874.

14. Fong HK, Hurley JB, Hopkins RS, Miake-Lye R, Johnson MS, Doolittle RF, Simon MI. Repetitive segmental structure of the transducin beta subunit: homology with the CDC4 gene and identification of related mRNAs. Proc Natl Acad Sci U S A. 1986;83(7):2162-6.

15. Hilton DJ, Richardson RT, Alexander WS, Viney EM, Willson TA, Sprigg NS, Starr R, Nicholson SE, Metcalf D, Nicola NA. Twenty proteins containing a C-terminal SOCS box form five structural classes. Proc Natl Acad Sci U S A. 1998;95(1):114-9.

16. Jia YY, Zhao JY, Li BL, Gao K, Song Y, Liu MY, Yang XJ, Xue Y, Wen AD, Shi L. miR-592/WSB1/HIF-1alpha axis inhibits glycolytic metabolism to decrease hepatocellular carcinoma growth. Oncotarget. 2016. doi:10.18632/ oncotarget.9135.

17. Kamura T, Burian D, Yan Q, Schmidt SL, Lane WS, Querido E, Branton PE, Shilatifard A, Conaway RC, Conaway JW. Muf1, a novel Elongin BC-interacting leucine-rich repeat protein that can assemble with Cul5 and Rbx1 to reconstitute a ubiquitin ligase. J Biol Chem. 2001;276(32):29748-53.

18. Kamura T, Sato S, Haque D, Liu L, Kaelin Jr WG, Conaway RC, Conaway JW. The Elongin BC complex interacts with the conserved SOCS-box motif present in members of the SOCS, ras, WD-40 repeat, and ankyrin repeat families. Genes Dev. 1998;12(24):3872-81.

19. Kazi JU, Kabir NN, Flores-Morales A, Ronnstrand L. SOCS proteins in regulation of receptor tyrosine kinase signaling. Cell Mol Life Sci. 2014; 71(17):3297-310.

20. Kehrer-Sawatzki H, Maier C, Moschgath E, Elgar G, Krone W. Characterization of three genes, AKAP84, BAW and WSB1, located 3 ' to the neurofibromatosis type 1 locus in Fugu rubripes. Gene. 1999;235(1-2):1-11.

21. Kibel A, Iliopoulos O, DeCaprio JA, Kaelin WG Jr. Binding of the von HippelLindau tumor suppressor protein to Elongin B and C. Science. 1995; 269(5229):1444-1446.

22. Kim JJ, Lee SB, Jang J, Yi SY, Kim SH, Han SA, Lee JM, Tong SY, Vincelette ND, Gao B, Yin P, Evans D, Choi DW, Qin B, Liu T, Zhang H, Deng M, Jen J, Zhang J, Wang L, Lou Z. WSB1 promotes tumor metastasis by inducing pVHL degradation. Genes Dev. 2015;29(21):2244-57.

23. Kim DH, Lee ST, Won HH, Kim S, Kim MJ, Kim HJ, Kim SH, Kim JW, Kim HJ, Kim YK, Sohn SK, Moon JH, Jung CW, Lipton JH. A genome-wide association study identifies novel loci associated with susceptibility to chronic myeloid leukemia. Blood. 2011;117(25):6906-11.

24. Linossi EM, Nicholson SE. The SOCS box-adapting proteins for ubiquitination and proteasomal degradation. IUBMB Life. 2012;64(4):316-23.

25. Lv W, Zhang Y, Wu Z, Chu L, Koide SS, Chen Y, Yan Y, Li Y. Identification of WSB1 gene as an important regulator in the development of zebrafish embryo during midblastula transition. Acta Biochim Biophys Sin. 2008; 40(6):478-88.

26. Moehlenbrink J, Bitomsky N, Hofmann TG. Hypoxia suppresses chemotherapeutic drug-induced p53 Serine 46 phosphorylation by triggering HIPK2 degradation. Cancer Lett. 2010;292(1):119-24.

27. Nara H, Onoda T, Rahman M, Araki A, Juliana FM, Tanaka N, Asao H. Regulation of interleukin-21 receptor expression and its signal transduction by WSB-2. Biochem Biophys Res Commun. 2010;392(2):171-7.

28. Nara H, Onoda T, Rahman M, Araki A, Juliana FM, Tanaka N, Asao H. WSB-1, a novel IL-21 receptor binding molecule, enhances the maturation of IL-21 receptor. Cell Immunol. 2011;269(1):54-9.

29. Nielsen KV, Ejlertsen B, Moller S, Jensen MB, Balslev E, Muller S, Knoop A, Mouridsen HT. Lack of independent prognostic and predictive value of centromere 17 copy number changes in breast cancer patients with known HER2 and TOP2A status. Mol Oncol. 2012;6(1):88-97.

30. Nucifora Jr FC, Nucifora LG, Ng CH, Arbez N, Guo Y, Roby E, Shani V, Engelender S, Wei D, Wang XF, Li T, Moore DJ, Pletnikova O, Troncoso JC, Sawa A, Dawson TM, Smith W, Lim KL, Ross CA. Ubiquitination via K27 and K29 chains signals aggregation and neuronal protection of LRRK2 by WSB1. Nat Commun. 2016:7:11792
31. Pollard PJ, Loenarz C, Mole D, McDonough MA, Gleadle JM, Schofield C, Ratcliffe PJ. Regulation of Jumonji-domain-containing histone demethylases by hypoxia-inducible factor (HIF)-1alpha. Biochem J. 2008;416(3):387-94.

32. Robinson $\mathrm{CM}$, Ohh M. The multifaceted von Hippel-Lindau tumour suppressor protein. FEBS Lett. 2014;588(16):2704-11.

33. Sagar GD, Gereben B, Callebaut I, Mornon JP, Zeold A, da Silva WS, Luongo C, Dentice M, Tente SM, Freitas BC, Harney JW, Zavacki AM, Bianco AC. Ubiquitination-induced conformational change within the deiodinase dimer is a switch regulating enzyme activity. Mol Cell Biol. 2007;27(13):4774-83.

34. Sar A, Ponjevic D, Nguyen M, Box AH, Demetrick DJ. Identification and characterization of demethylase JMJD1A as a gene upregulated in the human cellular response to hypoxia. Cell Tissue Res. 2009;337(2):223-34.

35. Sarraj MA, McClive PJ, Szczepny A, Daggag H, Loveland KL, Sinclair AH. Expression of Wsb2 in the developing and adult mouse testis. Reproduction. 2007:133(4):753-61.

36. Shichrur K, Feinberg-Gorenshtein G, Luria D, Ash S, Yaniv I, Avigad S. Potential role of WSB1 isoforms in growth and survival of neuroblastoma cells. Pediatr Res. 2014;75(4):482-6.

37. Staaf J, Jonsson G, Ringner M, Vallon-Christersson J, Grabau D, Arason A, Gunnarsson H, Agnarsson BA, Malmstrom PO, Johannsson OT, Loman N, Barkardottir RB, Borg A. High-resolution genomic and expression analyses of copy number alterations in HER2-amplified breast cancer. Breast Cancer Res. 2010;12(3).

38. Stirnimann CU, Petsalaki E, Russell RB, Muller CW. WD40 proteins propel cellular networks. Trends Biochem Sci. 2010;35(10):565-74.

39. Tong Y, Li QG, Xing TY, Zhang M, Zhang JJ, Xia Q. HIF1 regulates WSB-1 expression to promote hypoxia-induced chemoresistance in hepatocellular carcinoma cells. FEBS Lett. 2013:587(16):2530-5.

40. Vasiliauskas D, Hancock S, Stern CD. SWiP-1: novel SOCS box containing WD-protein regulated by signalling centres and by Shh during development. Mech Dev. 1999:82(1-2):79-94.

41. Villamil MA, Liang Q, Zhuang Z. The WD40-repeat protein-containing deubiquitinase complex: catalysis, regulation, and potential for therapeutic intervention. Cell Biochem Biophys. 2013;67(1):111-26.

42. Wall MA, Coleman DE, Lee E, Iniguez-Lluhi JA, Posner BA, Gilman AG, Sprang SR. The structure of the G protein heterotrimer Gi alpha 1 beta 1 gamma 2. Cell. 1995:83(6):1047-58.

43. Wan Y, Qu K, Zhang QC, Flynn RA, Manor O, Ouyang Z, Zhang J, Spitale RC, Snyder MP, Segal E, Chang HY. Landscape and variation of RNA secondary structure across the human transcriptome. Nature. 2014;505(7485):706-9.

44. Wang Y, Hu XJ, Zou XD, Wu XH, Ye ZQ, Wu YD. WDSPdb: a database for WD40-repeat proteins. 2014. Nucleic Acids Research

45. Wang Z, Liu P, Inuzuka H, Wei W. Roles of F-box proteins in cancer. Nat Rev Cancer. 2014:14(4):233-47.

46. Wellmann S, Bettkober M, Zelmer A, Seeger K, Faigle M, Eltzschig HK, Buhrer C. Hypoxia upregulates the histone demethylase JMJD1A via HIF-1. Biochem Biophys Res Commun. 2008;372(4):892-7.

47. Zavacki AM, Arrojo EDR, Freitas BC, Chung M, Harney JW, Egri $P$, Wittmann G, Fekete C, Gereben B, Bianco AC. The E3 ubiquitin ligase TEB4 mediates degradation of type 2 iodothyronine deiodinase. Mol Cell Biol. 2009;29(19):5339-47.

48. Zeold A, Pormuller L, Dentice M, Harney JW, Curcio-Morelli C, Tente SM, Bianco AC, Gereben B. Metabolic instability of type 2 deiodinase is transferable to stable proteins independently of subcellular localization. J Biol Chem. 2006;281(42):31538-43.

49. Zhang JG, Farley A, Nicholson SE, Willson TA, Zugaro LM, Simpson RJ, Moritz RL, Cary D, Richardson R, Hausmann G, Kile BJ, Kent SB, Alexander WS, Metcalf D, Hilton DJ, Nicola NA, Baca M. The conserved SOCS box motif in suppressors of cytokine signaling binds to elongins $B$ and $C$ and may couple bound proteins to proteasomal degradation. Proc Natl Acad Sci U S A. 1999:96(5):2071-6.

50. Zhao L, Mao Y, Zhao Y, Cao Y, Chen X. Role of multifaceted regulators in cancer glucose metabolism and their clinical significance. Oncotarget. 2016. 